\title{
Distinctive distribution of HPV genotypes in cervical cancers in multi-ethnic Suriname: implications for prevention and vaccination
}

\author{
M. G. GRUNBERG ${ }^{1}$, M. $\mathrm{CHAN}^{2}$ AND M. R. ADHIN ${ }^{3}$. \\ 1 'Prof. Dr. Paul C. Flu' Institute for Biomedical Sciences, Paramaribo, Suriname \\ ${ }^{2}$ Department of Pathology, Academic Hospital Paramaribo, Paramaribo, Suriname \\ ${ }^{3}$ Department of Biochemistry, Faculty of Medical Sciences, Anton de Kom Universiteit van Suriname, \\ Paramaribo, Suriname
}

Received 3 September 2015; Final revision 1 August 2016; Accepted 31 August 2016; first published online 20 October 2016

\section{SUMMARY}

Suriname is ranked as high-risk country for cervical cancer, but recent national data of HPV prevalence and distribution in cervical cancer is scarce. In a retrospective cross-sectional study, cervical cancer incidence, HPV prevalence and HPV-type-specific distribution were investigated in all cervical cancer cases $(n=111)$, diagnosed in two consecutive years. HPV presence and type-specific prevalence were determined in paraffin-embedded biopsies utilizing master-nested multiplex PCR assays, targeting 14 HPV types. The age-standardized incidence rate of cervical cancer was $22 \cdot 4 / 100000$ women, justifying revision of the current international ranking of Suriname. Eleven HPV types were detected, with the most common types in descending order of frequency: 16, 18, 45, 66, 58/52/35. HPV16 was predominant, although with markedly low presence (25\%). HPV16 or 18 infections were detected in $43 \%$ of the cases, while $28 \%$ were untyped, implicating a divergent HPV-type distribution in Suriname with significant variation in the prevalence of less common high-risk virus types and/or presence of HPV16 variants. HPV-type distribution differed between ethnic groups. A vaccination efficacy of just $28-30 \%$ was anticipated, next to an uneven vaccination impact in different ethnic groups, cautioning Suriname and other multi-ethnic countries to tailor the information presented to different ethnic communities.

Key words: Human papilloma virus (HPV), incidence, vaccine policy development.

\section{INTRODUCTION}

Cervical cancer (CC) is one of the most common cancers in women worldwide with the highest incidence rates in developing countries. More than 120 types of human papillomavirus (HPV) have been identified and classified according to their oncogenic potential [1]. Although considerable controversy exists about

\footnotetext{
* Author for correspondence: Dr M. R. Adhin, Faculty of Medical Sciences, Department of Biochemistry, Anton de Kom Universiteit van Suriname, Kernkampweg 5, Paramaribo, Suriname. (Email:m.adhin@uvs.edu)
}

the categorization of several low prevalent HPV types according to risk, the International Agency for Research on Cancer (IARC) has classified HPV16, 18, $31,33,35,39,45,51,52,56,58$ and 59 , as group 1 highrisk oncogenic types [1]. Meta-analyses have shown that in all regions of the world, the majority of CCs are due to infection with HPV16 or 18, the first and second most prevalent HPV types, respectively $[2,3]$. However, considerable inter- and intra-regional variations have been reported for the distribution and role of other common high-risk types, namely HPV31, 33, 45, 52, 58 and 35. Although some differences may be accounted for by heterogeneity in typing methods 
and/or insufficient representation of some regions, certain geographical differences have been reported consistently across all meta-analyses.

The proportion of invasive cervical cancer (ICC) cases harbouring HPV16/18 worldwide is highest in North America (76.4\%), followed by Europe (73.8\%) and Africa (70\%), while Asia (66.9\%) and South America (65\%) display a lower prevalence [4]. On the other hand, the prevalence of multiple infections is higher in developing countries in Africa (12.4\%) and South/Central America (11\%), than in countries in Asia (7.8\%), Europe $(6 \cdot 2 \%)$ and North America $(5 \cdot 1 \%)$ [4].

Most studies have focused on geographical differences in HPV-type prevalence but in the United States [5] and Malaysia [6] in-country differences in HPV prevalence across ethnic populations have also been reported. The relevance of HPV types other than HPV16/18 in CCs in specific regions and populations is especially relevant for targeted cancer prevention strategies and estimation of vaccination impact.

The two main approaches for $\mathrm{CC}$ prevention are HPV vaccination and oncogenic HPV detection during screening, allowing early treatment. Currently there is a bivalent and a quadrivalent HPV vaccine available. The impact of vaccination depends on the distribution of high-risk HPV types in a given population, since both vaccines target only high-risk types HPV16 and 18.

Suriname is a sparsely populated country in South America, where CC is among the leading causes of cancer-related deaths in women. The considerable contribution of HPV types other than HPV16/18 in CC in South America [16] and the multi-ethnic population of Suriname accentuated the need for detailed HPV data especially in the context of the initiation of a national vaccination programme. Since 2013, the government has introduced HPV vaccination for schoolgirls aged 10-12 years, through a school-based vaccination programme.

The aim of this study was to determine the CC incidence, HPV prevalence and type-specific distribution in $\mathrm{CC}$ in the multi-ethnic population of Suriname in a 2-year period, shortly before the first vaccinations started.

\section{METHODS}

\section{Study site}

Suriname is situated along the North Coast of South America with the majority of the population of
541638 persons (49.96\% male, 50.04\% female) [7] living in and around the capital Paramaribo. The population is highly diverse and consists of various distinct ethnic groups; $27 \cdot 4 \%$ Hindustani, $21 \cdot 7 \%$ Maroon (descendants of escaped West African slaves), 15.7\% Creole (descendants of slaves who lived in the city during slavery or mixed primarily with Dutch Europeans), 13.7\% Javanese, Amerindians (indigenous), Chinese, Caucasians (mostly descendants from Dutch and Portuguese Europeans) and others.

\section{Study population and sample collection}

Ethics approval was granted by the national Ethical Committee of the Ministry of Health (VG010-2012).

Archival, formalin-fixed paraffin-embedded biopsies of all women diagnosed with CC in Suriname in 2010 and $2011 \quad(n=111)$ were retrieved from the Pathology Department (Academic Hospital), the only Pathology facility in Suriname. These data therefore reflect the national situation. Samples from patients with a history of cervical conization or hysterectomy or with a diagnosis other than CC after confirmatory histological re-examination were excluded. From each formalin-fixed paraffin-embedded biopsy, $10-\mu \mathrm{m}$ sections of tissue were sliced, adhering to a strict protocol to prevent cross-contamination of samples. The first and last sections of the biopsies were re-examined by an external pathologist to confirm the histopathological diagnoses. The sections in between were stored for DNA testing.

\section{Laboratory testing}

DNA was extracted from two $10-\mu \mathrm{m}$ tissue sections with the QIAamp FFPE Tissue kit (Qiagen BV, The Netherlands) according to the manufacturer's protocol. The quality of extracted DNA was monitored through PCR amplification of the human $\beta$-globin gene. Specimens with a negative result were reextracted with three $10-\mu \mathrm{m}$ tissue sections. For samples remaining negative after two consecutive extractions, new sections were obtained from the paraffin block to repeat the extraction process.

HPV DNA was detected using a master-nested PCR combination utilizing the MY09/MY011 and GP05+/GP06+ primers [8]. HPV genotyping utilized a master E6/E7 PCR in combination with three multiplex PCRs [9], enabling the detection of 14 individual HPV types (high-risk types HPV16, 18, 31, 33, 35, 39, $45,52,56,58,59$, the possibly carcinogenic HPV66 
and low-risk types HPV6 and 11). Amplification products were detected on agarose or polyacrylamide gels after ethidium bromide staining. Negative and positive controls were included in each PCR run.

\section{Data collection and analysis}

Clinical records were traced in all hospitals and patients' information was anonymized. Patient characteristics, histopathological diagnosis and cancer type (squamous cell carcinoma/adenocarcinoma/clear cell carcinoma) were recorded.

Statistical analysis was performed using SPSS v. 21 (IBM Corp., USA). Because of the relatively small sample size in subgroups, differences between groups were analysed using non-parametric tests (MannWhitney test in continuous data and Fisher's exact test in proportions). For all tests $P<0.05$ was considered statistically significant.

\section{RESULTS}

In 2010 and 2011, a total of 111 patients were diagnosed in Suriname with ICC, reflecting an age-standardized incidence rate of CC of 22.4/100 000 women. The majority of the cases were histopathologically classified as squamous cell carcinomas (91\%), adenocarcinomas accounted for $9 \%$, while no rare histological variants were detected.

One patient had an incomplete clinical record and two tissue specimens could not be retrieved.

All retrieved biopsies were confirmed as ICC upon reexamination, although $7 \%$ were reclassified mostly as squamous cell carcinoma instead of adenocarcinoma.

DNA extraction of the retrieved biopsies $(n=109)$ was not successful for 13 samples $(12 \%)$, as attested by the PCR results, probably due to the inhibitory effect of remnants of paraffin, without significant variation in biopsies originating from 2010 and 2011. Samples with insufficient DNA yield were excluded. HPV DNA was detected in $91 \%$ of the valid samples.

General analysis of tumour type and analysis of age distribution was performed for all patients $(n=111)$, while analysis of HPV-type-specific distribution was performed on HPV-positive samples $(n=87)$. The HPV-type-specific distribution in CC cases presented in Table 1, shows 11 high-risk HPV types and the most common types, in descending order of frequency, were types $16,18,45,66$ and 58/52/35. The high-risk type HPV39 and low-risk types HPV6 and 11 were not detected in the CC samples. HPV16 was
Table 1. Distribution of HPV types in cervical cancer cases in Suriname (2010-2011)

\begin{tabular}{ll}
\hline \hline HPV type $(n=87)$ & $\%$ \\
\hline HPV16 & $25 \cdot 3$ \\
HPV18 & $17 \cdot 2$ \\
HPV45 & $12 \cdot 6$ \\
HPV66 & $4 \cdot 6$ \\
HPV35 & $3 \cdot 4$ \\
HPV52 & $3 \cdot 4$ \\
HPV58 & $3 \cdot 4$ \\
HPV31 & $1 \cdot 1$ \\
HPV33 & $1 \cdot 1$ \\
HPV56 & $1 \cdot 1$ \\
HPV59 & $1 \cdot 1$ \\
HPV39 & $0 \cdot 0$ \\
HPV6/11 & $0 \cdot 0$ \\
HPV X & $27 \cdot 6$ \\
Multiple types* & $2 \cdot 3$ \\
\hline \hline
\end{tabular}

* Double infections (multiple types) have been scored for each HPV type.

the most common type, with a presence of $25 \%$. The contribution of HPV16 and 18 amounted to $43 \%$. Worldwide, $87 \%$ of all CC cases are associated with the seven HPV types 16, 18, 45, 31, 33, 52 and 58 [10], while these high-risk HPV types account for just $64 \%$ of the CCs in this study.

The percentage of HPV positives not typed with the panel used, amounted to $28 \%$ (HPVX). The majority $(97 \cdot 7 \%)$ of the positive samples carried a single infection.

The HPV-type distribution seemed different for the two tumour types. Of the squamous cell tumours, the highest attribution was observed for HPV16, in contrast to the adenocarcinomas, mirroring the results from various other studies. However, the number of valid adenocarcinomas was rather small, precluding statistical analysis and therefore only overall data of ICC is discussed.

The mean age in the patient population $(n=111)$ was $53 \cdot 2$ years (range $27-91$ years). The mean age was lower in HPV-negative women $(52 \cdot 3$ years vs. $61 \cdot 2$ years, $P=0 \cdot 266$ ) than in HPV-positive women. The age distribution in the predominant HPV types is depicted in Figure 1.

The highest number of positive cases for HPV18 (53\%) and HPV16 (41\%) were noted in the 41-50 years age group. Women infected with HPV types 18 and 45 were on average 6 years younger at diagnosis than women with tumours associated with other 


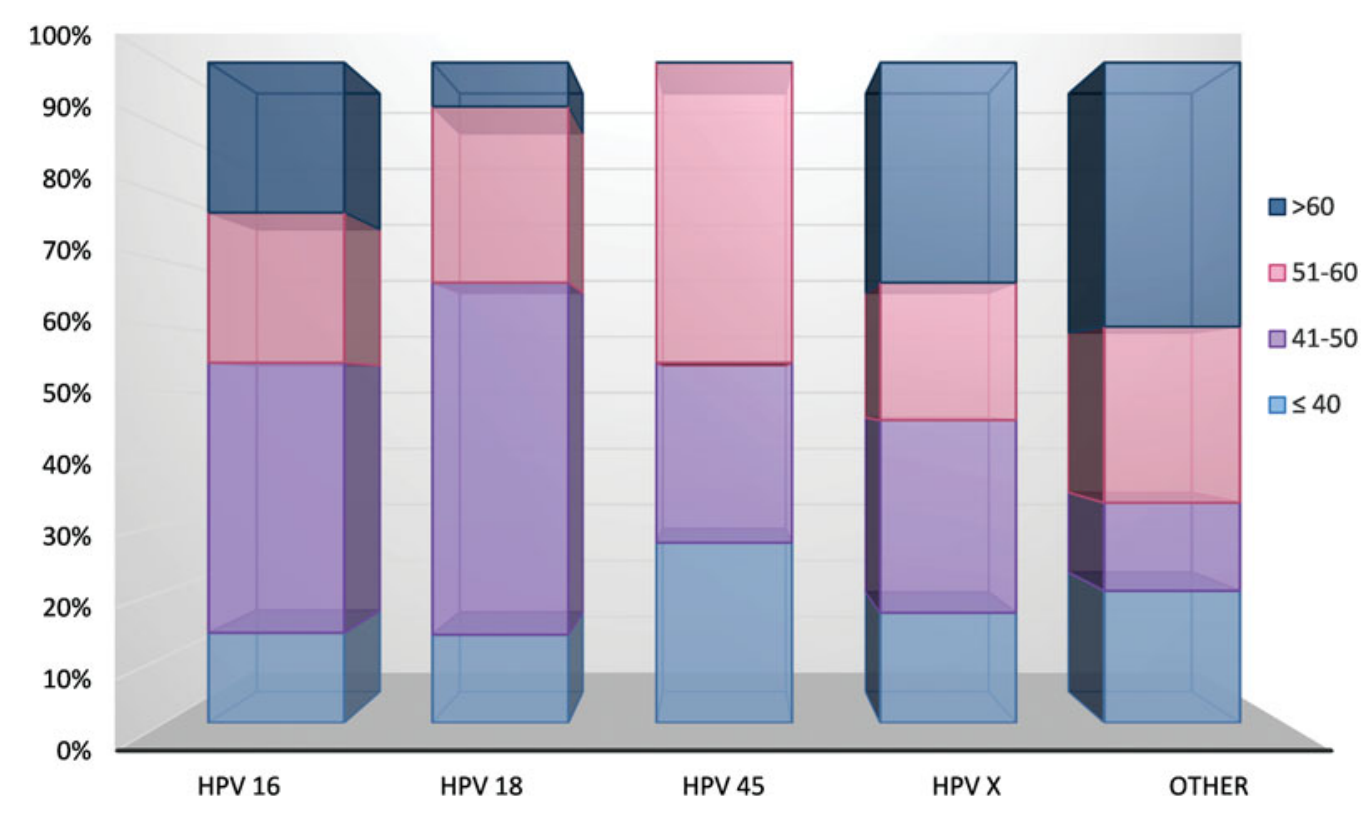

Fig. 1. Age distribution in the predominant HPV types. HPV occurrence in percentages for four age groups.

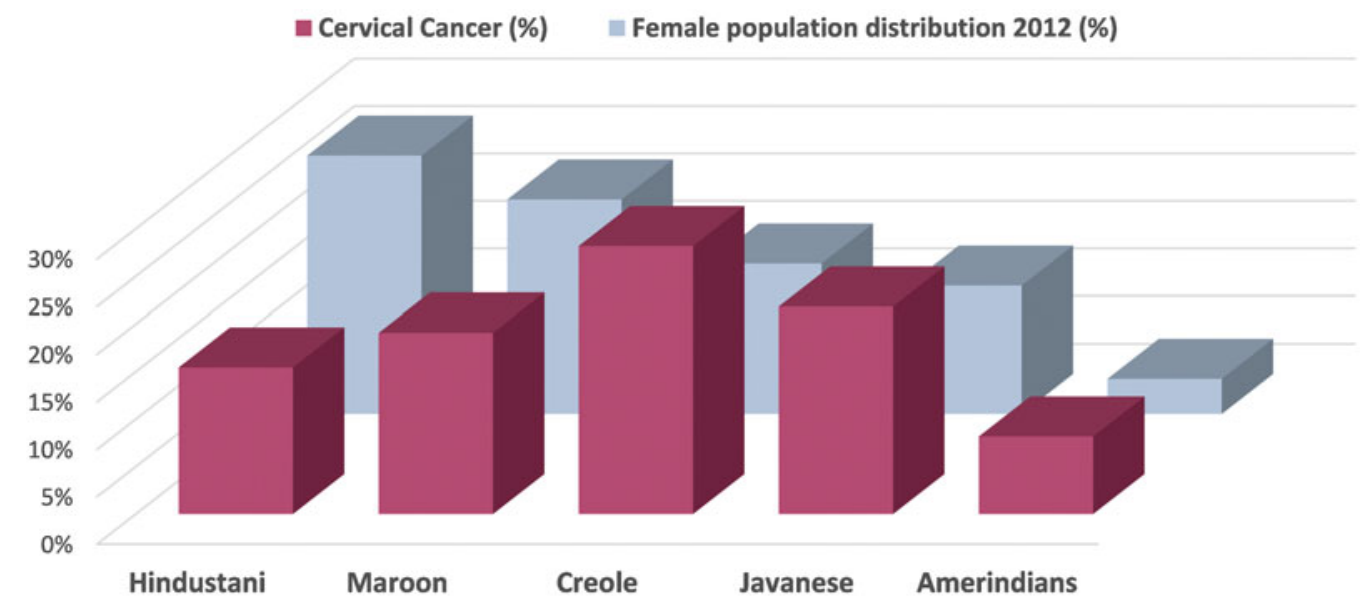

Fig. 2. Cervical cancer cases (\%) per ethnic group against the population data.

HPV types. The majority of HPV16, 18 and 45 infections were found in women aged $<50$ years, in contrast to the other types.

Differences in HPV prevalence were observed in ethnic groups with a very high prevalence in Chinese and Amerindians, a lower prevalence in Javanese, and the prevalence dropped below $90 \%$ in descendants from immigrants from India and persons of African descent.

$\mathrm{CC}$ incidence was also significantly different between the various ethnic groups and is depicted against the population data in Figure 2. CC is more prevalent in Creoles, Javanese and Amerindians than in the major ethnic groups, Hindustani and Maroons.
HPV distribution in the major ethnic groups is different as shown in Figure 3. Maroons displayed a higher percentage of HPV16 (50\%) compared to the other ethnic groups $(21 \%)(P=0 \cdot 038)$. Creoles displayed the highest genetic diversity with at least 10 different genotypes, whereas Maroons and Amerindians exhibited considerable less HPV genetic diversity.

\section{DISCUSSION}

\section{$\mathrm{CC}$ incidence}

The age-standardized incidence rate of CC in 2010/ 2011 was $22 \cdot 4 / 100000$ women, marking a decrease 


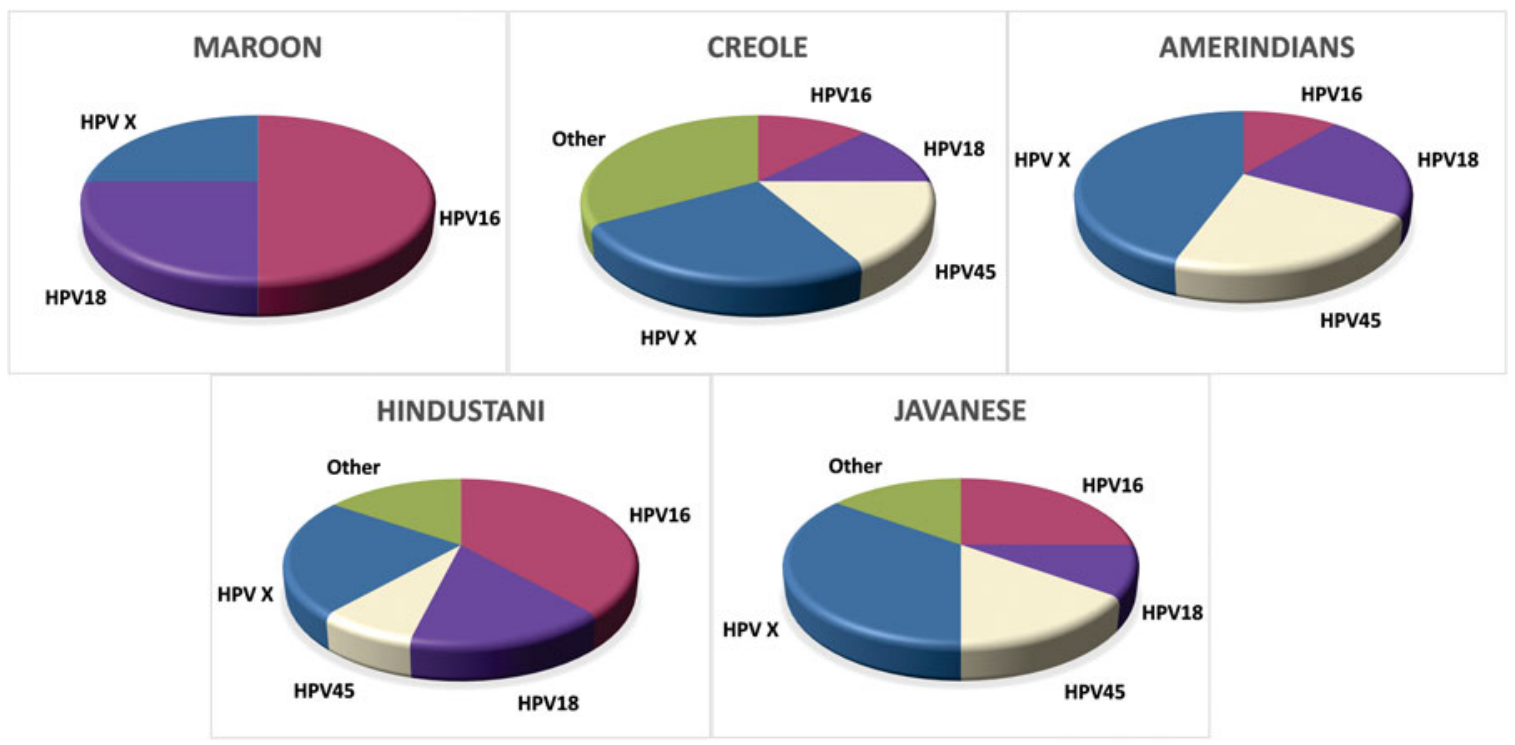

Fig. 3. HPV-type distribution in the major ethnic groups. 'Other' includes HPV types 31, 33, 35, 52, 56, 58, 59, 66. HPV X corresponds to untyped HPV positives.

in Suriname within 20 years $(26 \cdot 7 / 100000$ women) [11], probably due to improved measures for early detection of pre-cancerous stages. This incidence rate is not only considerably higher than the estimated global crude incidence rate (14.0), but also places Suriname higher than neighbouring Brazil (17.3) and overall South America (20.4). On the other hand, this incidence is lower than neighbouring countries, French Guiana (29.1) and Guyana (44.7) [12], and more importantly significantly lower than the IARC estimates based on national mortality estimates using modelled survival [13]. Consequently, Suriname should be removed from the top 20 list of countries with the highest incidence of CC [14].

\section{HPV overall prevalence and HPV-type prevalence}

The HPV prevalence of $91 \%$ in ICC in Suriname is in line with the overall global presence (89.9\%) [3].

A high diversity of HPV types was exhibited with 11 high-risk HPV types detected in patients with CC. The absence of low-risk types HPV6 and 11, concurs with their minimal presence $(0.9 \%)$ in cancers worldwide [3]. The seven most common HPV types, in descending order of frequency, were types 16, 18, 45, 66 and 58/52/ 35 . HPV16 and 18 are the first and second most prevalent types, corresponding with South American data and worldwide reports. However, the HPV genotype distribution in Suriname for the next most common types seems divergent from South America. HPV45 was the third most prominent type (13\%) similar to Africa, North America, and Western/Central Asia [3], while HPV31 as the third most common type in Central/South America (7.4\%) [10] and Europe, was underrepresented with just 1\%. HPV39, which seems almost entirely confined to Central/South America [15] was not even detected. A relatively minor contribution was noted for HPV33, which consistently ranks in the top five in Central and Latin America and in the United States [16, 17]. However, the third and fourth HPV types differ across regions and the amount of samples limited conclusions for less common HPV types.

Another interesting finding was the group 2B HPV66 type ranking ahead of HPV31 and 33, accentuating support for the reassessment of the carcinogenicity classification of HPV66, as was suggested in a recent study on biological activity of HPV66 in cancer tissue [18].

The predominance of HPV16 is consistent with other studies, but the HPV16 presence $(25 \%)$ was markedly lower than the overall prevalence of $57 \cdot 4 \%$ [10] ranging from $42 \%$ in Africa [19] to $53 \cdot 2 \%$ in Central/Latin America [16] and even 96\% in Greenland natives [20]. However, low HPV16 presence has been observed earlier, especially in countries in South America (Chile $32 \%$ ), Africa (Ghana 24\%) and Asia (Japan 29.6\%) [21-23]. A contributing factor to the low HPV16 presence could be the multiplex PCR assay, with primers originally designed and tested on a European population, which might be less suitable for HPV16 subtypes 
or intratypic variants circulating elsewhere. On the other hand, another study utilizing several primer sets still yielded a HPV16 prevalence of 38\% [24], still well below the worldwide prevalence. Either, HPV16 has a less prominent role in CC in Suriname or high genetic variation in HPV16 may have caused mismatched primer binding. This notion is supported by a comparative study between The Netherlands and Suriname, reporting a higher occurrence of nonEuropean HPV16 intratypic variants in Suriname [24]. Furthermore, the intratypic HPV16 variants reported for Suriname seemed distinctively different from Central and South America. Explanations could be related to the different immigration patterns, leading to the highly multi-ethnic population in Suriname with an extensive potential for genetic diversity.

HPV18 was the second most common type (17\%), consistent with international reports with an overall prevalence ranging from $13 \cdot 2 \%$ in Latin America and the Caribbean to $20 \%$ in Oceania [3, 16].

The joint contribution of HPV16 and 18 was significantly less frequent than in the rest of the world ( $43 \%$ vs. $70 \%, P<0.001$ ), due to the particularly low occurrence of HPV16.

The considerable differences implicated a divergent HPV genotype distribution in Suriname with a significant variation in the prevalence of some less common high-risk virus types and/or the presence of HPV16 variants. This premise is accentuated by the significantly higher percentage of unsubtyped cases than reported for Caribbean/Latin America (28\% vs. $10 \%$, $P<0.001)$. This result is most likely not due to the typing method, since the high percentage of untyped cases was corroborated in a recent study using a different method, a line probe assay targeting 25 different genotypes in Suriname, in which the unsubtyped cases of women visiting a STI clinic exceeded the persons infected with HPV16 [25]. The data on epidemiology and physiopathogenesis of non-HPV16/18 types, subtypes and HPV16 intratypic variants is limited, but these results indicate a substantial impact on the aetiology of ICC in Suriname.

Only $2 \cdot 3 \%$ of the samples harboured two HPV types, which is lower than the estimated $11 \cdot 2 \%$ multiple infections in worldwide meta-analyses [3]. On the other hand, considerable differences in multiple infections have been reported, ranging from $2.0 \%$ in Japan [23] up to $95 \%$ in Syria [26]. One should take into account that the occurrence of multiple types may have been underestimated, due to the use of a panel with 14 HPV types. This seems unlikely, as the same assay could detect $22 \%$ multiple infections including triple infections in a previous study conducted in pre-cancerous women in Suriname (our unpublished data). The observed difference in multiple infections emphasizes the differences in HPV prevalence in pre-cancer stages and ICC. Further support was derived from the low occurrence of HPV18 ( $2 \%$ in an earlier study carried out in pre-cancerous patients in Suriname) vs. 17\% HPV18 in this study $(P<0.001)$ and the predominance of HPV52 in women recruited at clinics in Suriname [25], not manifested as ICC. The current results substantiate the finding that HPV types that preferentially progress to ICC differ from HPV types in asymptomatic women or women with low- or high-grade lesions [17].

\section{HPV distribution in relation to age}

The mean age of women diagnosed with $\mathrm{CC}$ in Suriname $(53 \cdot 2$ years) was higher than in Central/ Latin America (50.7 years) and Asia (47.7 years) [27], which may indicate delayed diagnosis, but could also be explained with the lower proportion of HPV16 in Suriname, since women infected with HPV16/18 are younger at diagnosis than women infected with other types. The higher age-specific prevalence observed in Suriname therefore also supports the importance of HPV16 intratypic variants or HPV types other than HPV16/18. The age distribution in the predominant HPV types displayed considerable differences (Fig. 1).

Women infected with HPV types 16, 18 and 45 were on average 6 years younger at diagnosis than women with tumours associated with other HPV types, a trend also observed in studies in England and Mexico $[28,29]$. HPV45, the third most common type in Suriname, was not even detected in the $>60$ years age group, consistent with other studies [30]. The majority of HPV16, 18 and 45 infections occurred in women aged $<50$ years, with the reverse being true for the other types, in line with earlier observations [30].

\section{HPV presence in relation to ethnicity}

HPV prevalence was highest for Chinese and Amerindians, while the prevalence dropped to $95 \%$ in Javanese and $<90 \%$ in descendants of immigrants from India and persons of African descent. These results are in line with results from Malaysia where HPV prevalence was highest in Chinese (95.5\%), followed by Malays $(91.9 \%)$ and Indians $(80 \%)$ [6]. 
The comparison of $\mathrm{CC}$ occurrence in ethnic groups set against the population data in Figure 2 revealed that Creoles, Javanese and Amerindians were significantly more prone to $\mathrm{CC}$, while conversely $\mathrm{CC}$ was less prevalent in Hindustani and Maroons. The Javanese are also the major risk group in Suriname for hepatitis B infection [31]. On the other hand, this predominance is not displayed in the HIV prevalence [32], suggesting that rather than promiscuity, a genetic predisposition in Javanese may cause favouring of HPV virus persistence.

The pattern of HPV distribution in the major ethnic groups (Fig. 3) displayed considerable differences, as was also reported for multi-ethnic Malaysia [6]. Especially, the higher percentage of HPV16 (50\%) in Maroons compared to the other ethnic groups (21\%) $(P=0.047)$ was noteworthy. As could be expected from their secluded surroundings, the Maroons and the Amerindians, traditionally living in the interior, exhibited considerable less HPV genetic diversity than the Creoles with the highest variation of 10 HPV types. The absence of the less prevalent HPV types (other types) in the Maroons and Amerindians should be noted and the Maroons did not even harbour the third most common HPV45.

The higher occurrence of HPVX in Javanese and Amerindians coincided with their higher HPV positivity. This phenomenon could be explained since different types display variation in their oncogenic potential, inferring that HPVX either as other type, subtype or intratypic variant could also display a higher oncogenicity. Intratypic HPV16 variants have been reported with a transforming potential different from the HPV16 prototype [33].

Another interesting feature is the considerable difference between the ethnic groups in Suriname compared to their historical lineage. The HPV16 prevalence observed in Hindustani descendants from India, was $38 \%$, while a HPV16 prevalence of $74 \%$ has been reported for India [34]. On the other hand, the results for HPV18 prevalence were similar $(15 \%$ vs. 14\%). The Javanese (descendants from Java) have a HPV16 count of $25 \%$, while $34 \%$ has been reported for Jakarta (Java) [35]. However, one should take into consideration that both the Hindustani and Javanese have a considerable contribution of untyped cases, $23 \%$ and $35 \%$, respectively. The slave trade route can be traced back to the Ghana area and comparison of the prevalence of HPV16 between Maroons and people from Ghana shows an inverse relationship with only $24 \%$ HPV16 in Ghana [22] vs. $50 \%$ in
Maroons in Suriname. These results indicate that ethnic origin alone will not explain the disparities in HPV prevalence, although genetic predisposition can influence susceptibility to HPV infection and infection response. The differences in HPV prevalence in CC should be attributed to a complex interplay of multiple factors such as heritable factors, exposure, circulating HPV types, oncogenicity of specific HPV variants, environmental factors, cultural practices and also differential access to screening. Caution is warranted for the comparative analysis, because of substantial methodological variations in the different studies. Sequencing data will provide international comparability of results and more reliable recommendations for virological surveillance and for features required in new vaccines.

\section{Implications for HPV vaccination efficacy}

These data from a pre-vaccination period reflect the national situation of HPV-type distribution in confirmed CCs, the ultimate objective of cancer prevention programmes, and these results can therefore function as baseline to assess shifts in HPV genotype prevalence in Suriname after vaccination.

The demonstration that at least 11 different genital HPV types were associated with CC in Suriname has important implications for HPV vaccination as CC prevention strategy. The currently available vaccines Gardasil $^{\circledR}$ (Merck \& Co. Inc., USA) and Cervarix ${ }^{\circledR}$ (GSK, UK) protect against the high-risk types HPV16 and 18. The HPV vaccine efficacy for reducing CIN/2-3 lesions associated with HPV16 and 18 is more than $90 \%$. Consequently, it has been estimated that vaccination can markedly reduce the burden of $\mathrm{CC}$ up to $71 \%$ worldwide.

However, in Suriname, the reduction of the annual burden of $\mathrm{CC}$ with Gardasil vaccination will range from $28 \%$ to $30 \%$, with $70 \%$ vaccine coverage. This result is considerably lower than for South America, with a reduction of $55-69 \%(70 \%$ coverage) [36] and The Netherlands, with an estimated $47 \%$ (50\% vaccine coverage) [37]. It should be taken into account that the impact may be higher due to serological crossprotection against other types, since clinical vaccine trials were not designed to show efficacy in nonvaccine types. Moreover, some of the HPVX cases may be intratypic HPV16 variants with mutations prohibiting subtyping, but not necessarily evading immunological response, thus increasing anticipated vaccination impact. 
Second-generation HPV vaccines include a nonavalent vaccine, additionally targeting HPV types 31,33 , 45,52 , and 58. Use of the nonavalent vaccine in Suriname could reduce the $\mathrm{CC}$ burden with an additional $15 \%$, leading up to $45 \%$ in the case of $70 \%$ coverage, but would still not avert a substantial subset of CCs.

This anticipated low vaccination impact dictates that Suriname should implement additional preventive measures and provide sufficient information to communities to prevent misguided feelings of protection.

Cost-effectiveness analyses for continuation of vaccination should be performed also considering differences in vaccine impact per ethnic group. For instance, the higher percentage of HPV16 and 18 together in Maroons $(75 \%)$ compared to the other ethnic groups (38\%, $P=0.026)$, indicates that vaccination output in Maroons will surpass the impact in other ethnic groups.

Suriname and other multi-ethnic countries should tailor the information and measures presented to different ethnic communities based on the anticipated uneven impact in different ethnic groups. Furthermore, the substantial contribution of non-HPV16/18 types in ICC in Suriname highlights continued customized high-risk HPV screening as a prerequisite for $\mathrm{CC}$ reduction in these settings and the necessity for well-designed next-generation vaccines. These results add to the data challenging the impact of HPV vaccination for regions where the prevalence of HPV16/18 is less pronounced.

\section{ACKNOWLEDGEMENTS}

Research was conducted at the 'Prof. Dr. Paul C. Flu' Institute for Biomedical Sciences. We acknowledge the Ministry of Health (MOH) Suriname for facilitating this work. We also thank J. Kartowidjojo, J. Faerber, Sh. Pancham, M. Oron for the laboratory support and L. Nahar-van Venrooij for the statistical analysis.

This work was supported through the Ministry of Health of Suriname.

\section{DECLARATION OF INTEREST}

None.

\section{REFERENCES}

1. Bouvard V, et al. A review of human carcinogens - Part B: biological agents. Lancet Oncology 2009; 10: 321-322.

2. de Sanjose S, et al. Human papillomavirus genotype attribution in invasive cervical cancer: a retrospective cross-sectional worldwide study. Lancet Oncology 2010; 11: 1048-1056.

3. Li N, et al. Human papillomavirus type distribution in 30,848 invasive cervical cancers worldwide: variation by geographical region, histological type and year of publication. International Journal of Cancer 2011; 128: 927-935.

4. Smith JS, et al. Human papillomavirus type distribution in invasive cervical cancer and high-grade cervical lesions: A meta-analysis update. International Journal of Cancer 2007; 121: 621-632.

5. Hariri S, et al. Human papillomavirus genotypes in high-grade cervical lesions in the United States. Journal of Infectious Diseases 2012; 206: 1878-1886.

6. Hamzi Abdul Raub S, et al. Distribution of HPV genotypes in cervical cancer in multi- ethnic Malaysia. Asian Pacific Journal of Cancer Prevention 2014; 15: 651-656.

7. General Bureau of Statistics. The Eighth Population and Housing Census in Suriname (volume I): Demographic and Social Characteristics and Migration. Suriname, 2013.

8. Greer CE, Wheeler CM, Manos MM. Sample preparation and PCR amplification from paraffin-embedded tissues. PCR Methods and Applications 1994; 3: S113S122.

9. Sotlar K, et al. Detection and typing of human papillomavirus by E6 nested multiplex PCR. Journal of Clinical Microbiology 2004; 42: 3176-3184.

10. Muñoz N, et al. Against which human papillomavirus types shall we vaccinate and screen? The international perspective. International Journal of Cancer 2004; 111: 278-285.

11. Krul EJ, et al. Cervical carcinoma in Surinam. Incidence and staging of cervical carcinoma between 1989 and 1994. Cancer 1996; 77: 1329-1333.

12. Arbyn M, et al. Worldwide burden of cervical cancer in 2008. Annals of Oncology 2011; 22: 2675-2686.

13. GLOBOCAN 2012. International Agency for Research on Cancer (http://globocan.iarc.fr/). Accessed 28 May 2015.

14. World Cancer Research Fund International. Cervical cancer statistics (http://www.wcrf.org/int/cancer-factsfigures/data-specific-cancers/cervical-cancer-statistics). Accessed 16 June 2015.

15. Bosch FX, et al. Prevalence of human papillomavirus in cervical cancer: a worldwide perspective. Journal of the National Cancer Institute 1995; 87: 796-802.

16. Ciapponi A, et al. Type-specific HPV prevalence in cervical cancer and high-grade lesions in Latin America and the Caribbean: systematic review and meta-analysis. PLOS ONE 2011; 6: e25493.

17. Insinga RP, et al. A systematic review of the prevalence and attribution of human papillomavirus types among cervical, vaginal and vulvar pre-cancers and cancers in the United States. Cancer Epidemiology, Biomarkers \& Prevention 2008; 17: 1611-1622.

18. Halec G, et al. Biological activity of probable/possible high-risk human papillomavirus types in cervical cancer. International Journal of Cancer 2013; 132: 63-71. 
19. Ndiaye C, et al. Human papillomavirus distribution in invasive cervical carcinoma in sub-Saharan Africa: could HIV explain the differences? Tropical Medicine \& International Health 2012; 17: 1432-1440.

20. Sebbelov AM, et al. Comparison of human papillomavirus genotypes in archival cervical cancer specimens from Alaska natives, Greenland natives and Danish Caucasians. Microbes and Infection 2000; 2: 121-126.

21. Roa SJC, et al. Microsatellite instability and human papilloma virus genotypes in preneoplastic and neoplastic uterine cervix lesions. Revista Médica de Chile 2007; 135: $37-44$.

22. Attoh $\mathbf{S}$, et al. Human papilloma virus genotypes in Ghanaian women with cervical carcinoma. East African Medical Journal 2010; 87: 345-349.

23. Nagai Y, et al. Detection of human papillomavirus DNA in primary and metastatic lesions of carcinoma of the cervix in women from Okinawa, Japan. American Journal of Clinical Oncology 2001; 24: 160-166.

24. de Boer MA, et al. Human papillomavirus type 16 E6, E7, and L1 variants in cervical cancer in Indonesia, Suriname, and The Netherlands. Gynecologic Oncology 2004; 94: 488-494.

25. Geraets DT, et al. Cross-sectional study of genital carcinogenic HPV infections in Paramaribo, Suriname: prevalence and determinants in an ethnically diverse population of women in a pre-vaccination era. Sexually Transmitted Infections 2014; 90:627-633.

26. Moustafa AE Al, et al. High-risk HPVs and human carcinomas in the Syrian population. Frontiers in Oncology 2014; 4: 68 .

27. Alemany L, et al. Time trends of human papillomavirus types in invasive cervical cancer, from 1940 to 2007. International Journal of Cancer 2014; 135: 88-95.

28. Powell N, et al. Cervical cancers associated with human papillomavirus types 16, 18 and 45 are diagnosed in younger women than cancers associated with other types: a cross-sectional observational study in Wales and Scotland (UK). Journal of Clinical Virology 2013; 58: $571-574$.

29. Guardado-Estrada M, et al. The distribution of high-risk human papillomaviruses is different in young and old patients with cervical cancer. PLOS ONE. Published online: 8 October 2014. doi:10.1371/journal.pone.0109406.

30. Murillo R, et al. HPV prevalence in Colombian women with cervical cancer: Implications for vaccination in a developing country. Infectious Diseases in Obstetrics and Gynecology. Published online: 20 December 2009. doi:10.1155/2009/653598.

31. Bänffer JR, et al. The epidemiology of hepatitis B infections in a poly-ethnic population. American Journal of Epidemiology 1977; 106: 507-511.

32. PANCAP. UNGASS reports - Global AIDS response progress report Suriname 2010 (http://www.pancap.org/en/ component/jdownloads/viewdownload/50-ungass-reportsglobal-aids-response-progress-report/115-suriname-2010country-progress-report-en.html?Itemid=398). Accessed 17 June 2015.

33. Zehbe I, et al. Human papillomavirus 16 E6 variants are more prevalent in invasive cervical carcinoma than the prototype. Cancer Research 1998; 58: 829-833.

34. Bhatla $\mathbf{N}$, et al. Human papillomavirus type distribution in cervical cancer in Delhi, India. International Journal of Gynecological Pathology 2006; 25: 398-402.

35. De Boer MA, et al. Human papillomavirus type 18 variants: histopathology and E6/E7 polymorphisms in three countries. International Journal of Cancer 2005; 114: 422-425.

36. Goldie SJ, et al. Mathematical models of cervical cancer prevention in Latin America and the Caribbean. Vaccine 2008; 26 (Suppl. 11): L59-L72.

37. Bogaards JA, et al. Long-term impact of human papillomavirus vaccination on infection rates, cervical abnormalities, and cancer incidence. Epidemiology (Cambridge, Mass.) 2011; 22: 505-515. 